\title{
Ocorrência e conservação de Touit melanonotus (Aves, Psittacidae) no sul do Brasil
}

\author{
Marcelo A. V. Vallejos ${ }^{1}$, Ricardo Belmonte-Lopes ${ }^{2,3,4}$, Louri Klemann-Junior ${ }^{5}$, Marcos R. Bornschein ${ }^{2,4,5}$, \\ Leonardo R. Deconto ${ }^{1}$, Eduardo Carrano ${ }^{5,6,7}$ \& Carlos O. A. Gussoni ${ }^{7}$ \\ 1. Hori Consultoria Ambiental, Rua Cel. Temístocles de Souza Brasil, 311, 82520-210, Curitiba, PR, Brasil. (marcelo@hori.bio.br) \\ 2. Laboratório de Dinâmica Evolutiva e Sistemas Complexos, Departamento de Zoologia, Universidade Federal do Paraná, Centro Politécnico, Caixa Postal 19020, 81531-990, Curitiba, PR, Brasil. \\ Programa de Pós-Graduação em Zoologia, Departamento de Zoologia, Universidade Federal do Paraná, Centro Politécnico, Caixa Postal 19020, 81531-990, Curitiba, PR, Brasil. \\ 4. Mater Natura - Instituto de Estudos Ambientais, Rua Lamenha Lins, 1080, 80250-020, Curitiba, PR, Brasil. \\ 5. Programa de Pós-Graduação em Ecologia e Conservação, Universidade Federal do Paraná, Centro Politécnico, 81531-990, Curitiba, PR, Brasil. \\ 6. Curso de Biologia, Escola de Saúde e Biociências, Pontifícia Universidade Católica do Paraná, Curitiba, PR, Brasil; Comitê Brasileiro de Registros Ornitológicos (CBRO). \\ . Departamento de Zoologia, Universidade Estadual Paulista, campus de Rio Claro. Avenida 24-A, 1515, Bela Vista, 13506-900, Rio Claro, SP, Brasil.
}

\begin{abstract}
Occurrence and conservation of Touit melanonotus (Aves, Psittacidae) in southern Brazil. The Brown-backed Parrotlet [Touit melanonotus (Wied, 1820)] is a rare forest species restricted to the Brazilian Atlantic Forest, and is included in the endangered category for species threatened of extinction. As there is a lack of knowledge about the species' natural history and occurrence, new observations can provide helpful information for parrotlet conservation. Here we reevaluate the first record for the species and present new records for the state of Paraná (southern Brazil). The vocalizations previously attributed to T. melanonotus, and which were used to infer its occurrence in the state, were examined by spectrogram analysis. These were actually found to be vocalizations of the Red-capped Parrot [Pionopsita pileata (Scopoli, 1769)], representing a case of misidentification. Even so, the Brown-backed Parrotlet occurs with some regularity in the state of Paraná, as shown by 12 records spread through seven localities spanning the entire Paraná coast. The Brown-backed Parrotlet was recorded mostly in lowland areas, and as these forest types suffer intense pressures, conservation efforts aiming the species preservation should focus in the maintenance of these habitats.
\end{abstract}

KEYWORDS. Brown-backed Parrotlet, lowland forests, Paraná coast, vocalization.

RESUMO. O apuim-de-costas-pretas [Touit melanonotus (Wied, 1820)] é uma espécie florestal endêmica da Mata Atlântica Brasileira e de relevante interesse conservacionista. Trata-se de uma ave com poucas informações disponíveis acerca de sua história natural e distribuição geográfica e novas observações são importantes fontes de informação para auxiliar na conservação da espécie. Aqui reavaliamos o primeiro registro da espécie e apresentamos novas observações no estado do Paraná, sul do Brasil. As vocalizações atribuídas a T. melanonotus e que consubstanciaram sua ocorrência no estado foram examinadas com auxílio de espectrogramas e identificadas como vozes de Pionopsitta pileata (Scopoli, 1769). Não obstante, T. melanonotus ocorre com certa regularidade no Paraná, como indicado por novos registros da espécie em sete localidades distribuídas por todo o litoral do estado. Esta ave foi registrada principalmente em florestas de terras baixas e, uma vez que esta fisionomia sofre intensas pressões antrópicas, sugerimos que esforços de conservação da espécie devem priorizar a preservação desses hábitats.

PALAVRAS-CHAVE. Apuim-de-costas-pretas, floresta litorânea, litoral do Paraná, vocalização.

O apuim-de-costas-pretas, Touit melanonotus (Wied, 1820), é um psitacídeo florestal raro e endêmico da Floresta Atlântica, particularmente da área de endemismo da Serra do Mar (Cracraft, 1985; Morrone, 2001; Straube \& Di Giácomo, 2007). A espécie conta com poucos registros ao longo de toda sua distribuição geográfica, que abrange o sul do estado da Bahia e os estados do Espírito Santo, Minas Gerais, Rio de Janeiro e São Paulo (Forshaw, 1977; Sick \& Teixeira, 1979; Sick, 1997; Silveira, 2008; BirdLife INTERNATIONAL, 2011).

A raridade de T. melanonotus fez com que sua conservação fosse alvo de preocupação desde a década de 1940, quando se sugeriu que, possivelmente, a espécie fosse comum nas florestas costeiras de São Paulo, passando posteriormente a ser uma das mais raras em toda a faixa litorânea do Brasil (PINTo, 1946). Sick (1969), por outro lado, mencionou que a espécie poderia ser menos rara do que suposto até então e, posteriormente, alocou o táxon como de status desconhecido (SICK \& TEIXEIRA, 1979). Atualmente é avaliada como ameaçada na categoria em perigo, no âmbito global (IUCN, 2011), e vulnerável, na lista nacional (MMA, 2003).

Considerando a grande lacuna de conhecimento sobre a espécie, seja de informações sobre aspectos de história natural ou distribuição geográfica, novas informações referentes a este psitacídeo são de grande importância para sua conservação. No presente trabalho reavaliamos o suposto primeiro registro da espécie no sul do Brasil (BARNETT et al., 2004) e apresentamos novas informações sobre sua ocorrência e status de conservação na região.

\section{MATERIAL E MÉTODOS}

A análise qualitativa dos espectrogramas foi realizada considerando somente a modulação das vozes e o formato geral das notas. Avaliamos gravações disponíveis no acervo digital xeno-canto (www.xenocanto.org), sendo estas: gravação que consubstancia o registro mencionado por BARNETT et al. (2004) (MS 1025); vocalizações em voo de Pionopsitta pileata (Scopoli, 1769) (XC 85348) e Touit melanonotus (XC 85445). A comparação com $P$. pileata foi realizada devido à grande similaridade vocal entre estas espécies (vide Whitney et al., 2002 e Minns et al., 2009).

Todos os registros recentes foram efetuados independentemente pelos autores, em expedições 
realizadas entre os anos de 1997 e 2012 a vários pontos do litoral do estado. Os apontamentos são acompanhados de informações relevantes sobre os locais dos flagrantes, tamanho dos bandos e outros dados de história natural coligidos oportunamente na ocasião das observações. As localidades georreferenciadas foram inseridas em um mapa de modo a contextualizar os registros no âmbito estadual e a cobertura florestal remanescente. Os tipos florestais foram reconhecidos e designados conforme os critérios para a classificação da vegetação brasileira propostos pelo Projeto RADAMBRASIL (Veloso et al., 1991; IBGE, 1992).

\section{RESULTADOS E DISCUSSÃO}

Reavaliação do primeiro registro da espécie no Paraná. O primeiro registro publicado de T. melanonotus no estado do Paraná foi realizado na Serra da Graciosa (parte do conjunto da Serra do Mar; BARnetT et al., 2004), tendo sido documentado por uma gravação do autor-sênior. Contudo, nossas análises indicaram que a frequência do canto, o ritmo e a modulação das frases são condizentes com o observado em P. pileata (Figs. 1A e 1B) e, consequentemente, a primeira documentação atribuída a $T$. melanonotus no Paraná trata-se de um registro de $P$. pileata. Esta conclusão concorda com uma recente reavaliação da gravação MS 1025 , realizada por um dos autores-júnior da referida comunicação (MinNs, 2010).

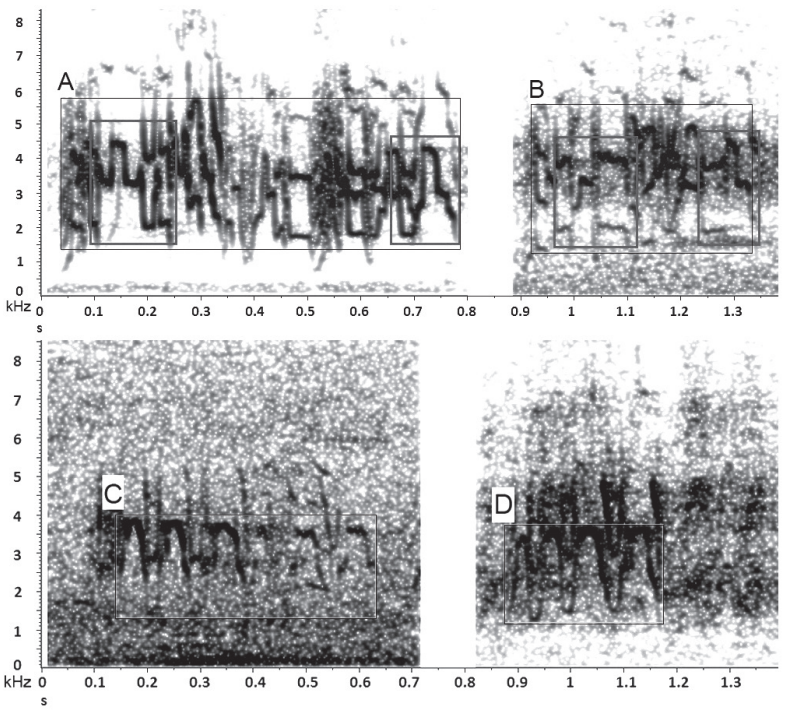

Fig. 1. Espectrogramas da vocalização de (A) Pionopsitta pileata (Scopoli, 1769) (gravação disponível em www.xeno-canto.org, código XC 85348); (B) vocalização atribuída a Touit melanonotus (Wied, 1820), mencionada em Barnett et al. (2004) (gravação disponível em www.xeno-canto.org, código MS 1025); (C) T. melanonotus obtida em Riozinho (Guaratuba, PR) por RB-L (gravação disponível em www.xeno-canto.org, código, XC 92140); (D) outra voz de T. melanonotus (gravação disponível em www.xeno-canto.org, código XC 85445). Imagens reduzidas em uma escala de $10,5 \mathrm{kHz}(8 \mathrm{kHz}$ na figura) no programa Raven 1.4, janela tipo Blackmann, de tamanho 1.024 e sobreposição de $96 \%$. Em destaque menor em "B" evidenciam-se semelhanças na modulação e frequência de dois trechos das frases.
Novas informações sobre Touit melanonotus no Paraná. Ao todo foram realizados 12 registros da espécie no estado, todos concentrados na vertente leste da Serra do Mar, no litoral (Fig. 2, Tab. I). O primeiro registro foi realizado por E. C., P. Scherer-Neto e M. Wasilewski em agosto de 1997 (citado em SCHERER-Neto et al. [2011] como 1996) na estrada para o Descoberto, município de Guaratuba, quando cinco indivíduos foram observados em área de floresta ombrófila densa de terras baixas secundária deslocando-se rapidamente em voo acima das árvores.

Quase nove anos após, COAG e RB-L registraram este psitacídeo em março de 2006 em Riozinho, município de Guaratuba. Uma gravação foi obtida de espécimes que sobrevoavam uma área de floresta ombrófila densa de terras baixas, consistindo na primeira documentação da ocorrência da espécie no estado do Paraná. A identidade da espécie foi confirmada após análises da gravação (Figs. 1C e 1D), haja vista que os indivíduos não foram visualizados nesta oportunidade. Outro registro foi obtido pelos mesmos autores no mesmo mês e ano, onde 12 indivíduos foram observados voando sobre uma estrada entre plantios de Pinus sp. contíguos a áreas florestadas, nas proximidades do registro vocal supracitado.

Após dois anos, em outubro de 2008, EC, LKJ e P. Scherer-Neto avistaram um pequeno grupo com seis indivíduos voando e vocalizando sobre uma área de floresta ombrófila densa submontana próxima à vila de Castelhanos, município de São José dos Pinhais. No ano seguinte, a espécie foi registrada por EC e LKJ em fevereiro de 2009 (citado em SchereR-Neto et al. [2011] como 2002) no município de Guaratuba, sendo observado um grupo com aproximadamente 20 indivíduos vocalizando em voo, os quais pousaram em meio a uma área de floresta ombrófila densa submontana. Ainda no mesmo ano, T. melanonotus foi observado em duas ocasiões na localidade de Porto de Cima, município de Morretes: o primeiro contato foi realizado em abril por MAVV, T. Molin e S. Baijuk, quando um grupo de seis indivíduos foi observado primeiramente em voo, vocalizando intensamente, e posteriormente pousados em Archontophoenix cunninghamii H.Wendl. \& Drude (Arecaceae) de uma propriedade particular; o segundo registro da espécie na mesma localidade foi em 1 de maio, por MAVV e LRD com um grupo de oito aves avistado sobrevoando a mesma propriedade, também vocalizando intensamente.

No ano seguinte, em outubro, T. melanonotus foi novamente registrado em Riozinho, município de Guaratuba. No dia 28, MRB registrou grupos da espécie em voo. Um dia após, MRB, RB-L, B.L. Reinert e D.D. Sobotka observaram um grupo de cerca de seis indivíduos pousados em Psidium guajava L. (Myrtaceae) junto a uma casa, obtendo-se registros fotográficos de três indivíduos (Fig. 3). As aves forrageavam isoladamente e em silêncio, alimentando-se de frutos de Struthanthus 


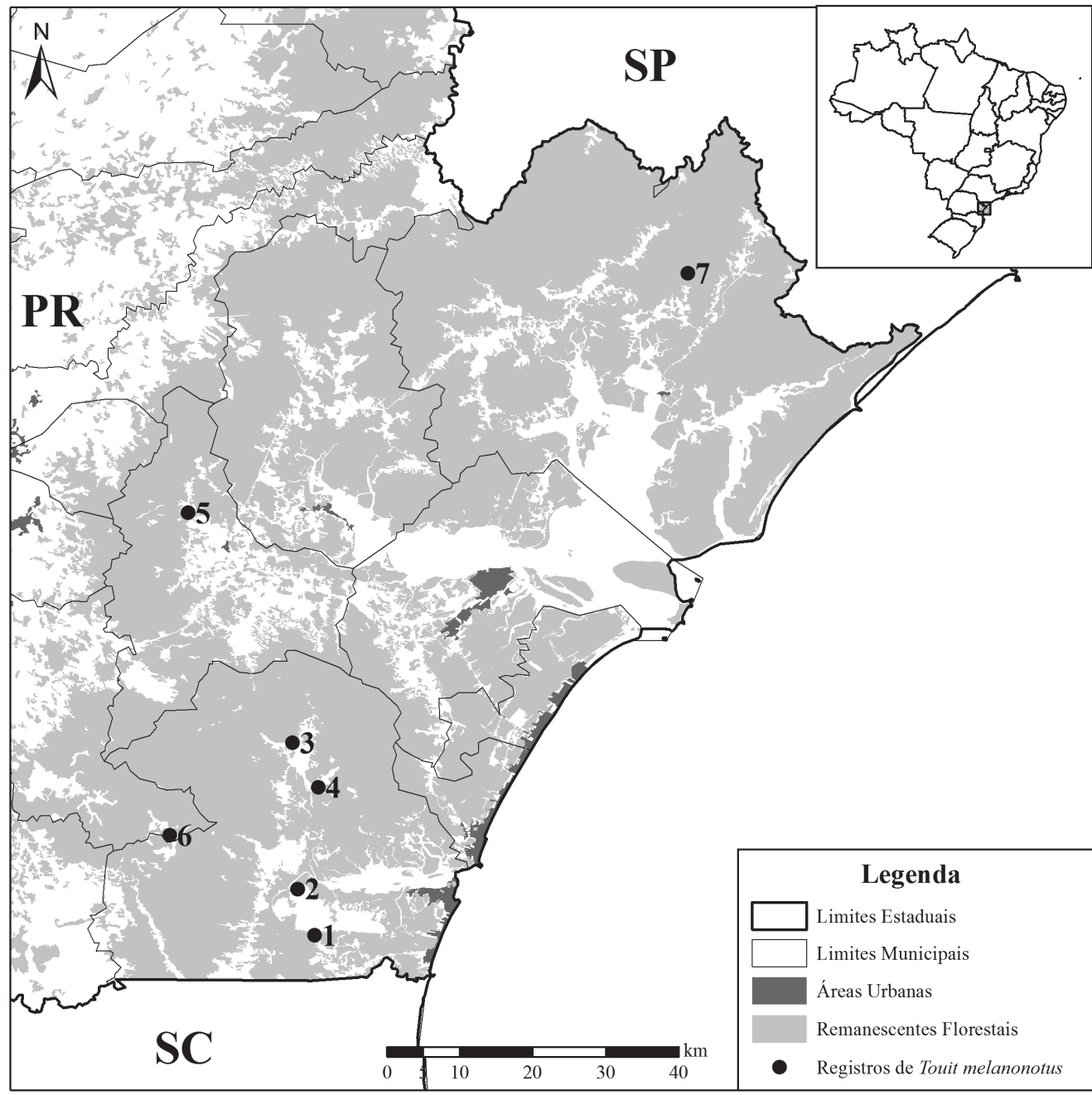

Fig. 2. Localidades de registro de Touit melanonotus (Wied, 1820) no estado do Paraná: 1, estrada para o Descoberto; 2, Riozinho; 3, Limeira; 4, Reserva Bicudinho-do-brejo; 5, Porto de Cima; 6, Castelhanos; 7, Reserva Natural Salto Morato.

Tab. I. Detalhamento dos registros de Touit melanonotus (Wied, 1820) no estado do Paraná, Brasil. Datas seguidas por “*” indicam que o registro é unicamente auditivo.

\begin{tabular}{|c|c|c|c|c|c|c|}
\hline $\mathrm{N}^{\mathrm{o}}$ & Município & Localidade & \multicolumn{2}{|c|}{ Coordenadas } & Datas & $\mathrm{N}^{\mathrm{o}}$ de indivíduos \\
\hline 1 & Guaratuba & Estrada para o Descoberto & $25^{\circ} 55^{\prime} 45^{\prime \prime} \mathrm{S}$ & $48^{\circ} 43^{\prime} 43^{\prime \prime} \mathrm{W}$ & 08.1997 & 5 \\
\hline \multirow{5}{*}{2} & \multirow{5}{*}{ Guaratuba } & \multirow{5}{*}{ Riozinho } & \multirow{5}{*}{$25^{\circ} 52^{\prime} 32^{\prime \prime} \mathrm{S}$} & \multirow{5}{*}{$48^{\circ} 44^{\prime} 53^{\prime \prime} \mathrm{W}$} & $05.03 .2006^{*}$ & $?$ \\
\hline & & & & & 08.03 .2006 & 12 \\
\hline & & & & & 29.10 .2010 & 6 \\
\hline & & & & & 30.10 .2010 & 2 \\
\hline & & & & & 09.03 .2012 & Cerca de 15 \\
\hline 3 & Guaratuba & Limeira & $25^{\circ} 42^{\prime} 20^{\prime \prime} \mathrm{S}$ & $48^{\circ} 45^{\prime} 14^{\prime \prime} \mathrm{W}$ & 21.02 .2009 & 20 \\
\hline 4 & Guaratuba & Reserva Bicudinho-do-brejo & $25^{\circ} 45^{\prime} 26^{\prime \prime} \mathrm{S}$ & $48^{\circ} 43^{\prime} 28^{\prime \prime} \mathrm{W}$ & 16.02 a 16.03 .2012 & $?$ \\
\hline \multirow{2}{*}{5} & \multirow{2}{*}{ Morretes } & \multirow{2}{*}{ Porto de Cima } & \multirow{2}{*}{$25^{\circ} 26^{\prime} 15^{\prime \prime} \mathrm{S}$} & \multirow{2}{*}{$48^{\circ} 52^{\prime} 33^{\prime \prime} \mathrm{W}$} & 18.04 .2009 & 6 \\
\hline & & & & & 01.05 .2009 & 8 \\
\hline 6 & São José dos Pinhais & Castelhanos & $25^{\circ} 48^{\prime} 46^{\prime \prime} \mathrm{S}$ & $48^{\circ} 53^{\prime} 49^{\prime \prime} \mathrm{W}$ & 10.10.2008 & 6 \\
\hline 7 & Guaraqueçaba & Reserva Natural Salto Morato & $25^{\circ} 11^{\prime} 04^{\prime \prime} \mathrm{S}$ & $48^{\circ} 17^{\prime} 56^{\prime \prime} \mathrm{W}$ & $22.10 .2011^{*}$ & ? \\
\hline
\end{tabular}

vulgaris Mart. (Loranthaceae). No dia 30 um bando foi gravado vocalizando em voo por MRB, e dois foram avistados por RB-L pousados em uma palmeira próxima à borda de uma área secundária de floresta ombrófila densa de terras baixas, emitindo uma vocalização distinta daquela emitida em voo e também das gravações disponíveis (WhitNeY et al., 2002; Minns et al., 2009; acervo digital xeno-canto).
Em outubro de 2011, um grupo com cerca de 30 indivíduos foi registrado sobrevoando ruidosamente uma área de floresta ombrófila densa submontana na Reserva Natural Salto Morato, município de Guaraqueçaba (F.C. Straube, com. pess.).

Finalmente, entre fevereiro e março de 2012, diversos contatos foram realizados por COAG e MRB com grupos vocalizando em voo na Reserva Bicudinho- 


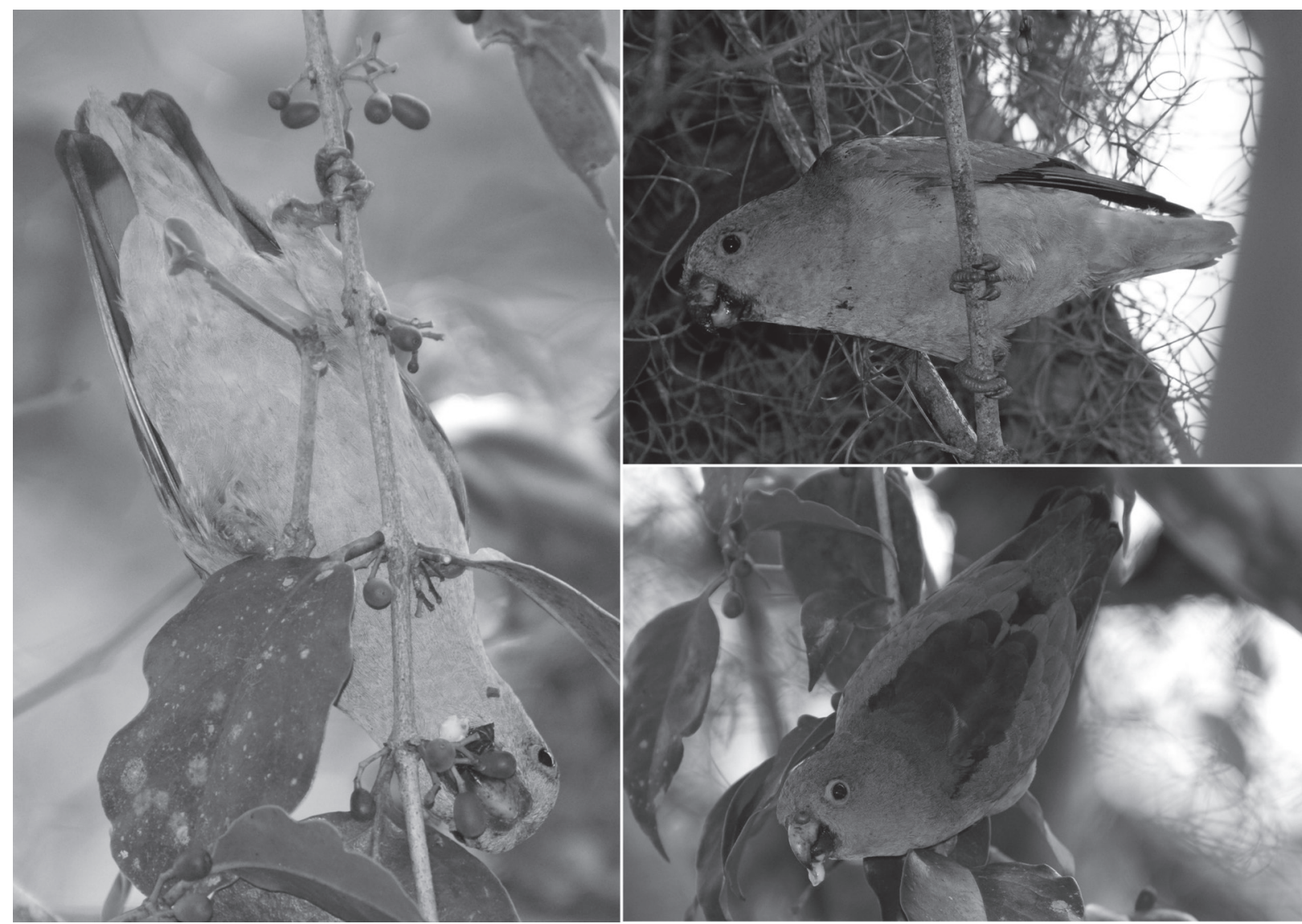

Fig. 3. Três indivíduos diferentes de Touit melanonotus (Wied, 1820) alimentando-se de frutos de Struthanthus vulgaris (Loranthaceae) em Riozinho, município de Guaratuba (PR). Fotos: Ricardo Belmonte-Lopes

do-brejo. E ainda, em Riozinho, COAG observou cerca de 15 indivíduos em março de 2012. Em todos os contatos os bandos apenas sobrevoavam o local, às vezes a baixa altura.

Estes registros ampliam a distribuição conhecida da espécie em cerca de $100 \mathrm{~km}$ para o sul de seu limite austral (Ilha do Cardoso; WegE \& Long, 1995), embora sua presença no Paraná fosse esperada. O limite meridional de ocorrência da espécie também contempla o litoral norte de Santa Catarina (EC, dados inéditos), que apresenta continuidade florestal com o litoral sul do Paraná, especialmente entre os municípios de Guaratuba (Paraná), Garuva e Itapoá (Santa Catarina), região onde se encontram os maiores remanescentes de floresta ombrófila densa de terras baixas do litoral sul do Paraná e Santa Catarina.

Uma vez que a biologia de $T$. melanonotus é pouco conhecida, os padrões de ocupação das matas paranaenses não são claros, podendo sua presença no estado ser atribuída tanto a padrões migratórios ainda incógnitos, colonização recente da região ou mesmo à simples falta de observações. Mesmo considerando o elevado esforço amostral despendido na região por diversos observadores e ornitólogos (STRAUBE \& SCHERER-Neto, 2001), esta última possibilidade não pode ser descartada, tendo em vista a raridade natural da espécie e a difícil identificação de sua voz em campo.
A maior parte dos registros da espécie foi realizada em áreas de floresta ombrófila densa de terras baixas, sendo essa uma das fácies vegetacionais cuja integridade é das mais ameaçadas (Sтотz et al. 1996). Esse tipo florestal continua sendo suprimido no litoral paranaense devido à formação de pastagens e cultivos de arroz e banana, assim como pelo crescimento urbano desordenado, a exemplo do verificado nas Áreas de Proteção Ambiental de Guaratuba (MRB, observ. pess.) e Guaraqueçaba (EC, observ. pess.). Destacam-se ainda como potenciais ameaças grandes projetos de infraestrutura, como a instalação do novo traçado da BR-101 entre a cidade de Garuva (SC) e a BR 277, no município de Morretes (PR), novos ramais rodoviários ligando a BR-277 às cidades de Paranaguá (PR) e Pontal do Sul (PR) e nova linha férrea. Há outros inúmeros empreendimentos de menor abrangência, mas que em conjunto também podem afetar grandes áreas dessa formação florestal ainda em bom estado de conservação. Considerando o fato dessa formação ser a menos protegida por Unidades de Conservação de Proteção Integral nos estados do Paraná e Santa Catarina, e também o grau de ameaça de T. melanonotus, a conservação da espécie dependerá de efetivos esforços para interromper a perda de hábitat, sua principal causa de ameaça. 
Agradecimentos. Alguns dos registros da espécie em Guaratuba foram efetuados durante fases de campo dos projetos 0682/20052 e 0911/20112 apoiados pela Fundação Grupo Boticário de Proteção à Natureza. RB-L é apoiado por bolsa de doutorado do Conselho Nacional de Desenvolvimento Científico e Tecnológico (CNPq/MCT 141823/2011-9); COAG é bolsista de doutorado da Fundação de Amparo à Pesquisa do Estado de São Paulo (FAPESP 2010/09707-1) e MRB da Coordenação de Aperfeiçoamento de Pessoal de Nível Superior (CAPES).

\section{REFÊRENCIAS}

Barnett, J. M.; Minns, J.; Kirwan, G. M. \& Remold, H. 2004. Informações adicionais sobre as aves dos estados do Paraná, Santa Catarina e Rio Grande do Sul. Ararajuba 12(1):55-58.

BIRDLIFE INTERNATIONAL. 2011. Species factsheet: Touit melanonotus. Disponível em <http://www.birdlife.org/datazone>. Acesso em: 29.06.2011.

Cracraft, J. 1985. Historical biogeography and patterns of differentiation within the South American avifauna: areas of endemism. Ornithological Monographs 36:49-84.

Forshaw, J. M. 1977. Parrots of the World. Neptune, T. F. H. Publications. 584p.

IBGE. 1992. Manuais técnicos em geociências: manual técnico da vegetação brasileira. Rio de Janeiro, Instituto Brasileiro de Geografia e Estatística. 92p.

IUCN. 2011 The IUCN Red List of Threatened Species. The World Conservation Union. Disponível em $<$ http://www.iucnredlist.org $>$. Acesso em: 23.11.2011.

Minns, J. 2010. Comentário on-line. Disponível em < www.xeno-canto. org/discussion.php?snd nr=1025>. Acesso em: 15.12.2011.

Minns, J.; Buzzetti, D.; Albano, C.; Grosset, A.; Whittaker, A. \& PARRINI, R. 2009. Aves do Brasil: vozes e fotografias, v. 1 -Floresta Atlântica, Cerrado, Caatinga, Pantanal, Campos Sulinos e Costa. Vinhedo, Avis Brasilis Editora, 1 DVD-Rom.

MMA - Ministério Do Meio Ambiente. 2003. Lista nacional das espécies da fauna brasileira ameaçadas de extinção. Brasília,
Ministério do Meio Ambiente. Disponível em < http://www.mma. gov.br>. Acesso em: 20.10.2012.

Morrone, J. J. 2001. Biogeografía de América Latina y el Caribe. Zaragoza, M\&T Manuales y Teses SEA, v.3. 148p.

Pinto, O. M. de O. 1946. Aves brasileiras da família dos papagaios. Relatório Anual do Instituto Botânico:126-129.

Scherer-Neto, P.; Straube, F. C.; Carrano, E. \& Urben-Filho, A 2011. Lista das aves do Paraná. Hori Cadernos Técnicos $n^{\circ} 2$. Curitiba, Hori Consultoria Ambiental. 130 p.

SICK, H. 1969. Aves brasileiras ameaçadas de extinção e noções gerais de conservação de aves no Brasil. Anais da Academia Brasileira de Ciências 41(suppl):71-93.

1997. Ornitologia Brasileira. 2ed. Rio de Janeiro, Nova Fronteira. 912p.

SiCK, H. \& TEIXEIRA, D. M. 1979. Notas sobre aves brasileiras raras ou ameaçadas de extinção. Rio de Janeiro, Publicações Avulsas do Museu Nacional n. 62. 39 p.

SilveIRA, L. F. 2008. Touitmelanonota.In:Machado,A. B. M.; Drummond, G. M. \& PAGLIA,A. P. eds. Livro vermelho da fauna brasileira ameaçada de extinção. Brasília, Fundação Biodiversitas, v.2, p. 485-486.

Stotz, D. F.; Fitzpatrick, J. W.; Parker III, T. A. \& Moskovits D. K. 1996. Neotropical Birds: ecology and conservation. Chicago, University of Chicago Press. 478p.

Straube, F. C. \& Di GiÁcomo, A. 2007. A avifauna das regiões subtropical e temperada do Neotrópico: desafios biogeográficos. Ciência \& Ambiente 35:137-166.

Straube, F. C. \& Scherer-Neto, P. 2001. História da ornitologia no Paraná. In: Straube, F. C. ed. Ornitologia sem fronteiras. Curitiba, Fundação O Boticário de Proteção à Natureza, p. 43-116.

Veloso, H. P.; Rangel-Filho, A. L. R. \& Lima, J. C. A. 1991. Classificação da vegetação brasileira, adaptada a um sistema universal. Rio de Janeiro, Fundação Instituto Brasileiro de Geografia e Estatística. 123 p.

Wege, D. C. \& Long, A. J. 1995. Key areas for threatened birds in the Neotropics. Cambridge, BirdLife International, series n. 5. 312p

Whitney, B. M.; Parker III, T. A.; Budney, G. F.; Munn, C. A. \& BrA DBury, J. W. 2002. Voices of New World Parrots. Ithaca, Cornell Lab of Ornithology, 3 CD-Rom. 